Please do not remove this page

RMIT

UNIVERSITY

\title{
Frontiers in diffraction unlimited optical methods for spin manipulation, magnetic field sensing and imaging using diamond nitrogen vacancy defects
}

Castelletto, Stefania; Li, Xiangping; Gu, Min

https://researchrepository.rmit.edu.au/esploro/outputs/9921858802901341/filesAndLinks?institution=61RMIT_INST\&index=null

Castelletto, S., Li, X., \& Gu, M. (2012). Frontiers in diffraction unlimited optical methods for spin manipulation, magnetic field sensing and imaging using diamond nitrogen vacancy defects. Nanophotonics, 1(2), 139-153. https://doi.org/10.1515/nanoph-2012-0001

Document Version: Published Version

Published Version: https://doi.org/10.1515/nanoph-2012-0001

Repository homepage: https://researchrepository.rmit.edu.au

(C) 2012 Science Wise Publishing and De Gruyter

Downloaded On 2023/04/27 00:52:15 +1000 
Thank you for downloading this document from the RMIT Research Repository.

The RMIT Research Repository is an open access database showcasing the research outputs of RMIT University researchers.

RMIT Research Repository: http://researchbank.rmit.edu.au/

\section{Citation:}

Castelletto, S, Li, X and Gu, M 2012, 'Frontiers in diffraction unlimited optical methods for spin manipulation, magnetic field sensing and imaging using diamond nitrogen vacancy defects', Nanophotonics, vol. 1, pp. 139-153.

See this record in the RMIT Research Repository at:

http://researchbank.rmit.edu.au/view/rmit:21939

Version: Published Version

Copyright Statement: ㄷ 2012 Science Wise Publishing and De Gruyter

Link to Published Version:

http://dx.doi.org/10.1515/nanoph-2012-0001 
The final publication is available at www.degruyter.com 


\section{Frontiers in diffraction unlimited optical methods for spin manipulation, magnetic field sensing and imaging using diamond nitrogen vacancy defects}

\author{
Stefania Castelletto ${ }^{1, *}$, Xiangping $\mathrm{Li}^{2}$ and $\mathrm{Min} \mathbf{G u}^{2}$ \\ ${ }^{1}$ Faculty of Science, Department of Physics and Astronomy, \\ Macquarie University, Sydney, NSW 2109, Australia, \\ e-mail: stefania.castelletto@mq.edu.au \\ ${ }^{2}$ Faculty of Engineering and Industrial Science, Centre for \\ Micro-Photonics, Swinburne University of Technology, \\ Hawthorn, VIC 3122, Australia \\ * Corresponding author
}

\begin{abstract}
The nitrogen vacancy defect centre in diamond has attracted intense research interest owing to their appealing optical and electronic properties, which have laid the ground for new approaches for diffraction unlimited optical methods. In particular, the optical detected magnetic resonance of the electron spin of nitrogen vacancy centre at room temperature underpins many areas in nanophotonics, spintronics and quantum optics. This article reviews the recent development of superresolution imaging and sensing nanoscopy based on this fascinating defect centre in diamond. These breakthroughs are presently indicating a new class of nanoscale sensors of tiny magnetic and electric fields at room temperature, as well as emerging fluorescent and magnetic probes for next generation nanoscopy and all-optical spin recording.
\end{abstract}

Keywords: nanodiamonds; super-resolution; optical and spin defects in diamond.

\section{Introduction}

Detecting spin of a single electron and nuclear spin is central in several fields of science including spintronics, quantum information and biology. Several techniques were successfully developed during the last decade including electrical and force based detection of spin states associated with individual atoms or molecules. Although the above-mentioned approaches are able to image and monitor dynamics of single spin states, they require low temperature operation and high vacuum conditions. Optical techniques that will be described in this review are unique owing to the possibility of detecting the dynamics of the single electron and nuclear spin under ambient conditions, and to associate the spin properties for implementing imaging methods to achieve sub-diffraction resolution. During the past few years nitrogen-vacancy (NV) centre has emerged as promising candidate for a number of applications ranging from high spatial resolution imaging [1-4], magnetometry [5-7] and quantum memories [8-10]. The negative charged state exhibits a strong dipole-allowed optical transition with a zero-phonon line (ZPL) at around $1.945 \mathrm{eV}$ (637 nm) [11] which allows for the photoluminescence based detection of single defect centres at room as well as cryogenic temperature. The photoluminescence intensity is strongly modulated by the $\mathrm{S}=1$ ground state electron spin. As a consequence optically detected electron spin resonance has been demonstrated to be single defects in bulk and nano-crystals [12].

At low temperature, the optical transitions of the NV centres become very narrow and can be coherently manipulated, allowing for spin-photon entanglement generation [13] for quantum communication and all optical control. A detailed understanding of the properties of this defect is critical for many of these applications. Several studies have addressed this issue both experimentally $[14,15]$ and theoretically [16]. Furthermore, other atom-like defects can potentially be engineered in diamond [17] and other materials [18] with similar or perhaps better properties suitable for the desired applications.

In Section 2 the physical and electronic properties of the NV centre will be reviewed, while in Section 3 the present synthesis methods of the defect in bulk diamond and nanodiamonds will be described. In Section 4, the optical spin manipulation methods such as optically detected magnetic resonance, Rabi oscillation and spin-echo measurements will be reviewed, that brought to the main recent research outcomes of NV in diamond. In Section 5, optical properties of NV centres such as photochromism, two-photon absorption and nonlinearity will be discussed. In Section 6, application of NV centres in nanodiamonds and bulk diamond in magnetic field sensing and super-resolution magnetic field imaging will be presented. In Section 7, optical nanoscopy implemented used NV centres will be reviewed in details, specifically stimulated emission depletion microscopy, ground state and dark state depletion microscope, reversable saturable optical fluorescence methods as well as sub-diffraction spin manipulation methods will be reviewed. Section 8 concludes this review article highlighting relevant experiments here described useful to access NV centres future deployment and to better understand the relevance of similar findings in other centres in diamond and related materials.

\section{Physical structure and electronic levels of the nitrogen vacancy centre}

Nitrogen-vacancy (NV) centres in diamond have been studied for many decades using a variety of techniques. Recently, 
there has been renewed interest in NV centres as a physical system for quantum information science in the solid state [19]. The NV centre is an attractive qubit candidate because it behaves like a trapped molecule in the diamond lattice: it has strong optical transitions, and an electron spin degree of freedom. In this section, we consider the basic structure of the NV centres and present experimental techniques used to probe its spin and optical transitions. The negative charged NV centre is a point defect in the diamond lattice. The model of the NV centre consists of a substitutional nitrogen atom adjacent to a vacancy in diamond (Figure 1A). The NV centre has a strong optical transition with a zero phonon line (ZPL) at $1.945 \mathrm{eV}$ $(637 \mathrm{~nm})$ accompanied by a vibronic band at higher energy in absorption and lower energy in emission. Detailed analysis of the ZPL revealed that the centre has trigonal $\mathrm{C}_{3 v}$ symmetry, unlike atoms in free space, whose electronic states are governed by their rotational invariance. The physical structure of this defect, and the symmetries associated with it, determine the nature of its electronic states and the dipole-allowed transitions between them. Two charge states of this defect, neutral $\mathrm{NV}^{0}$ and negative $\mathrm{NV}^{-}$, are known from spectroscopic studies using optical absorption $[11,20]$ photoluminescence (PL) (Figure 1B) [21], electron paramagnetic resonance (EPR) [22] and optically detected magnetic resonance (ODMR) [12], which can be viewed as a hybrid of PL and EPR. A nitrogen atom has five valence electrons. Three of them covalently bond to the carbon atoms and two remain nonbonded. The vacancy has three unpaired electrons. Two of them make a quasi covalent bond and one remains unpaired. The $\mathrm{NV}^{0}$ thus has one unpaired electron and is paramagnetic. However, despite extensive efforts, electron paramagnetic resonance signals from $\mathrm{NV}^{0}$ avoided detection for decades until optical excitation has been used to detect $\mathrm{NV}^{0}$ into the EPR-detectable excited state [23]. In the negative charge state $\mathrm{NV}^{-}$, the extra electron is located at the vacancy site forming a spin $\mathrm{S}=1$ pair with one of the vacancy electrons. As in $\mathrm{NV}^{0}$, the vacancy electrons are "exchanging roles" preserving the overall trigonal symmetry. The neutral state has not yet been explored for spin manipulations. The energy level structure of the NV- centre was established over 20 years by combining optical, electron paramagnetic resonance and theoretical results, as shown in Figure 2. In particular, several theoretical works have been done, using the Linear Combination of Atomic Orbitals approach, to build the electronic orbitals to describe the possible quantum states, looking at the NV centre as a molecule. Moreover, group theory results are used, to take into account the symmetry of the diamond crystal, and so the symmetry of the NV itself. The energy levels are labeled according to the group theory and considering the $\mathrm{C}_{3 v}$ symmetry group of NV, where A indicates a symmetric level (orbital singlet) while $\mathrm{E}$ is a not symmetric level (orbital doublet). A recent work from Maze et al. [27] shows ab-initio calculation to determine the order of singlet and triplet state in the ground state. The numbers 3 in ${ }^{3} \mathrm{~A}$ and 1 in ${ }^{1} \mathrm{~A}$ represent the number of allowable $m_{s}$ spin states, or the spin multiplicity, which range from $-\mathrm{s}$ to $\mathrm{s}$ for a total of $2 \mathrm{~s}+1$ possible states. If $\mathrm{S}=1, \mathrm{~m}_{\mathrm{s}}$ can be $-1,0$, or 1 . It has been established that the ground electronic state is a spin triplet ${ }^{3} \mathrm{~A}_{2}$. This triplet is itself split by spin-spin interactions, yielding one $\mathrm{Sz}$ or $\mathrm{m}_{s}=0$ state with $\mathrm{A}_{1}$ character and two $\mathrm{m}_{s}= \pm 1$ states [10] with $\mathrm{E}$ character, which are 2.87 $\mathrm{GHz}$ higher in energy. Together with the $637 \mathrm{~nm}$ ZPL, the $2.87 \mathrm{GHz}$ zero-field ground-state allows the identification of a defect in diamond as an NV centres. The $S=1$ ground state structure has been well established by many experiments, while the excited state structure has only recently been fully understood by resonant excitation at low temperature [15, 25]. The excited state ${ }^{3} \mathrm{E}$ is also a spin triplet. Besides, the ${ }^{3} \mathrm{E}$ excited state is an orbital doublet, in which degeneracy is lifted by non-axial strain into two orbital branches, Ex and Ey, each orbital branch being formed by three spin states Sx, Sy, and Sz. Thermal population transfer between the different orbitals in the excited state prevents from having a polarized light emission associated with $\mathrm{NV}$ at room temperature and for broadband excitation, associate to dynamic Jahn-Teller [26]. As optical transitions ${ }^{3} A_{2} \rightarrow{ }^{3} E$ are spin conserving, excitation spectra of single NV colour centres can show six resonant lines, corresponding to transitions between identical spin sublevels. The averaged energy splitting between $\mathrm{Sz}$ and
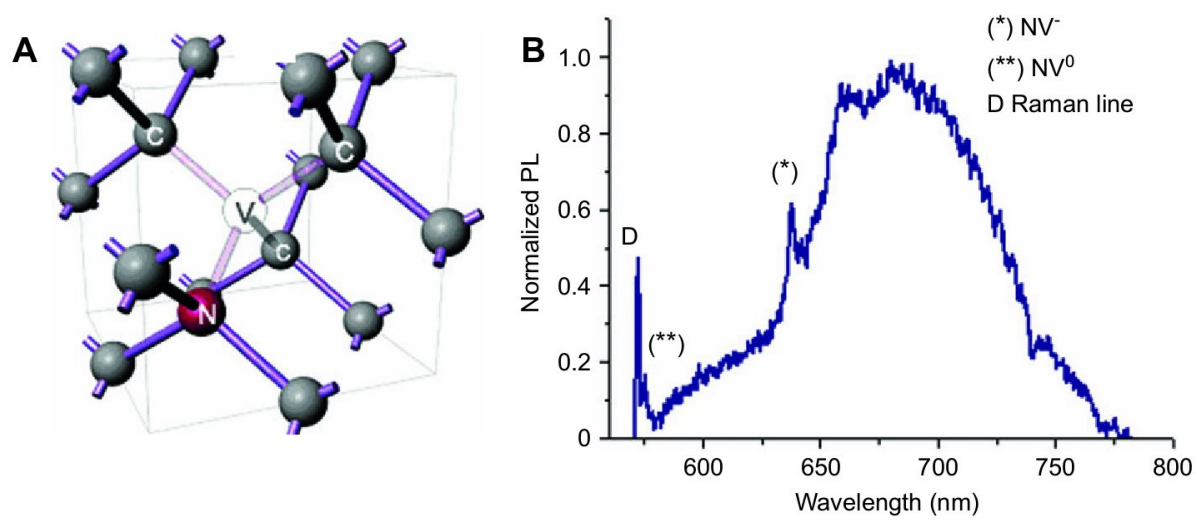

Figure 1 The NV centre. (A) Crystallographic model of the NV centre in diamond, consisting of a substitutional nitrogen (shown in red) adjacent to a vacancy (V). (B) Room temperature PL spectrum showing the ZPLs of the neutral (575 $\mathrm{nm}$ ) and the negatively charged (637 nm) NV centre with pronounced and wide phonon side bands at lower energy side of each ZPL. 

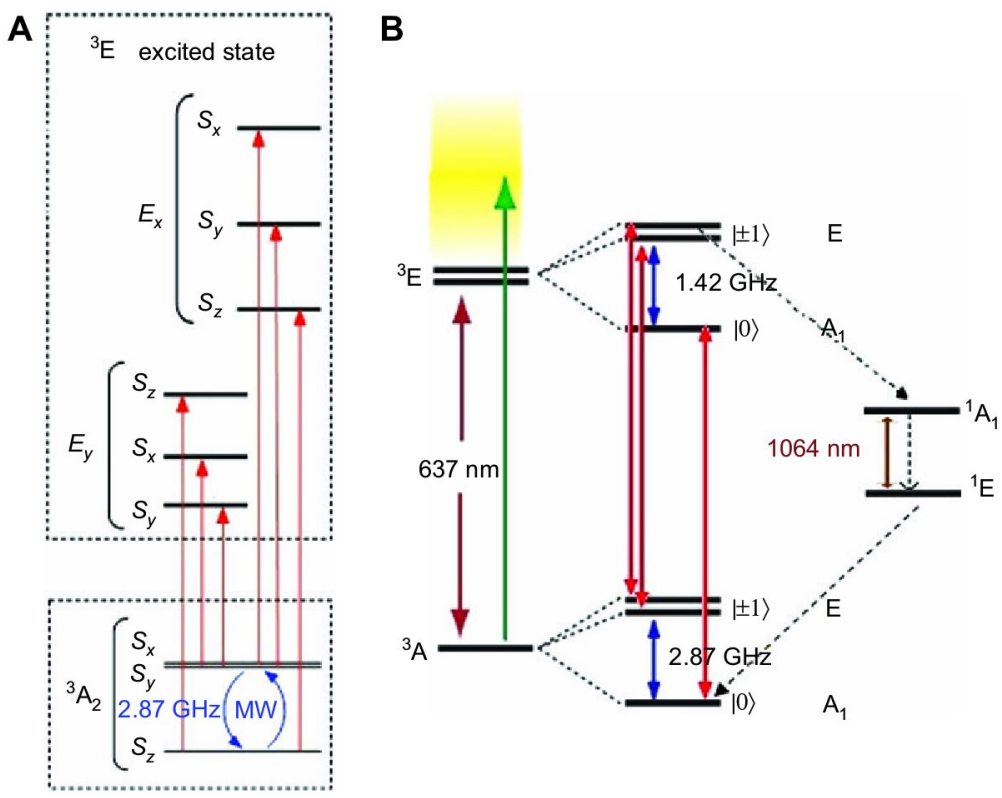

Figure 2 The NV centre. (A) Detailed energy-level diagram of a single NV defect ground and excited state [24]. (B) Room temperature energy levels with radiative transition (solid red) and non-radiative spin selective transition (dashed lines). Microwave frequency separation of the zero field in the ground state and excited state are also indicated in blue [25]. The excited state polarization emission properties are strongly dependent on the temperature and strain [26].

Sx, Sy excited-state spin sublevels is then only determined by the parameter Des, which has a value of $1.42 \mathrm{GHz}$, in low strain condition. The order of other energy levels is still under debate, but it is now well established that at least one metastable state ${ }^{1} \mathrm{~A}$ lies between the ground and excited triplet state. This metastable state plays a crucial role in spin dynamics of the NV colour centre. Indeed, whereas the optical transitions ${ }^{3} A \rightarrow{ }^{3} E$ are spin conserving, non-radiative inter-system crossing transitions to the metastable state ${ }^{3} E \rightarrow^{1} A$ are strongly spin selective as the shelving rate from the $\mathrm{m}_{s}=0$ sublevel is much smaller than those from $\mathrm{m}_{s}= \pm 1$. Conversely, the metastable state decays preferentially towards the ground state $m_{s}=0$ sublevel, leading to a strong spin polarization into $\mathrm{m}_{s}=0$ after a few optical excitation-emission cycles. The metastable state has been recently demonstrated to possess an absorption in the infrared at $1064 \mathrm{~nm}$ [28].

\section{Material and defects synthesis}

An NV centre is a defect typically incorporated during diamond growth in chemical vapour deposition (CVD) or high temperature high pressure synthesis (HPHT). However, a common method to increase NV concentration and formation, is by irradiating the material with high energy particles, creating vacancies in the diamond, and subsequent annealing in vacuum $\left(<10^{-4}\right.$ mbar $)$ for $1 \mathrm{~h}$ with temperature ranges from $700^{\circ} \mathrm{C}$ to $1000^{\circ} \mathrm{C}$. At this temperature vacancies become mobile and the formation probability of NV centres increases. A wide range of high-energy particles are suitable for such irradiation, including electrons, protons, neutrons, ions, and gamma photons. Irradiation produces lattice vacancies, which are a part of $\mathrm{NV}^{-}$centres. Those vacancies are immobile at room temperature, and annealing is required to move them. The dose of the irradiation dictates the density of centres created in the material, however the starting material with intrinsic impurities needs to be taken into account before performing irradiation. Typical irradiation doses to achieve single emission are quite low, $10^{10}$ ions $/ \mathrm{cm}^{2}$ until $10^{14}$ ions/ $\mathrm{cm}^{2}$, while higher doses such as $10^{15}$ are used for high concentration of centres mostly for biological applications. The type of irradiation to create colour centres in diamond to be detected by luminescence spectroscopy strongly depends on the starting material, differentiated from monolithic crystal or nanodiamonds (NDs).

Both bulk and NDs can be produced in a technological process. Synthetic diamond is also widely known as HPHT diamond or CVD diamond. CVD is also used for creating nanosized scale diamond and to control the diamond doping by impurities diffusion during growth; however, regardless, this method appears more effcient than irradiation doping [29], it remains a challenge to fully control the type of impurities as well as the size of the nanoparticles down to few nanometers and in particular to create NDs with very high purity.

A third method, known as detonation synthesis, entered the diamond market in the late 1990s. In this process, nanometersized diamond grains are created in a detonation of carboncontaining explosives. Detonation nanodiamonds (DNDs) are currently the focus of present intense research, due to their promises to extend the use of this material in domains that are exclusively dominated by quantum dots and single molecules. DNDs ( $5 \mathrm{~nm}$ in diameter) can be formed by detonating certain carbon-containing explosives in a metal chamber. During the explosion, the pressure and temperature in the chamber 
become high enough to convert the carbon of the explosives into diamond. Being immersed in water, the chamber cools rapidly after the explosion, suppressing conversion of newly produced diamond into more stable graphite. The product is always rich in graphite and other nondiamond carbon forms and requires prolonged boiling in hot nitric acid (about 1 day at $250^{\circ} \mathrm{C}$ ) to dissolve them. For its use to be pursued in fundamental physics experiments or bio-imaging, long procedures involving acid boiling and refluxing for days and centrifuge or oxidation performed by air heating at $450^{\circ} \mathrm{C}$, have been performed to enhance the emission of $\mathrm{NV}$ centres naturally present in the material. Other methods, treating graphite with high-power ultrasound or long-pulsed laser ablation, and recently directly created in aerogel [30] have become attractive for the preparation of NDs; they have been demonstrated in the laboratory, but currently there is no application in photoluminescence spectroscopy.

Diamonds are separated into four types, according to nitrogen impurities concentration: type Ia, type Ib, type IIa, and type IIb, as it is in natural diamond classifications. The most commonly used for the applications of this review are type Ib, type IIa and ultra-pure diamonds.

This type of diamond control can only be determined in monolithic diamond synthetised by HPHP or CVD methods, while NDs can be synthetised starting from this type of monolithic material. Type $\mathrm{Ib}$ contains up to $0.05 \%$ (500 ppm) of nitrogen, but the impurities are more diffuse, the atoms are dispersed throughout the crystal in isolated sites. Type II diamonds have almost no natural counterpart since nitrogen impurities cannot be measured by absorption spectroscopy. However they are detectable by luminescence and therefore relevant for these applications. Type IIa contain $<100 \mathrm{ppm}$ nitrogen impurities, while boron concentration is also present, typically less $0.5 \mathrm{ppm}$. Finally, recently ultra pure diamond has been synthesized containing $<5$ ppb nitrogen concentration and $<1 \mathrm{ppb}$ of boron. The synthesis of this material has been a relevant breakthrough for the here presented applications, since the absence of impurities carrying paramagnetic properties is a key for controlling parasitic magnetic field, that can be sensed by an NV centre. An important approach for creating NV defects in electronic grade diamond where nitrogen content is very low is based on direct irradiation of nitrogen ions at various energy and fluences. The nitrogen implantation introduces both nitrogen atoms and vacancies, which combine upon annealing. The nitrogen implantation into low $\mathrm{N}$ diamond is a suitable method to fabricate low density NV for qubits in diamond [31]. For scalable architecture requiring array of NV centres applications, one needs an accurate positioning of the NV centres as well as a high probability of creation. These two conditions have not yet been simultaneously fulfilled. The implantation experiments have shown that two major issues related to the creation of colour centres need to be addressed: (a) spatial resolution and (b) yield of creation of color centres. The implantation technique has intrinsic limitation on defects positioning accuracy arising from straggling of nitrogen in diamond lattice during implantation. Low (a few $\mathrm{keV}$ ) energy is necessary for achieving a positioning accuracy in the nanometer range, however the yield of conversion of nitrogen to NV centres during annealing is low in this case (a few percents, due to an insuffcient number of vacancies, to surface trapping of vacancies during annealing, and to higher $\mathrm{NV}^{0} / \mathrm{NV}^{-}$ratio). A striking recent demonstration [32] shows that high spatial accuracy of NV implantation can be realised using novel implantation/detection technology. Since dopants are buried into the diamond lattice, scanning probe techniques like AFM or STM cannot be used to characterise the created arrays. Nanometer scale mapping of single implanted atoms can instead be done using far-field optical super-resolution microscopy. The possibility to produce NDs of this purity in size $<10 \mathrm{~nm}$ is the key technological challenge of the moment for the here presented applications. The first fluorescent NV in NDs at $10 \mathrm{~nm}$ has been reported by Tisler and coworkers [33], where type Ib NDs from HPHT synthesis, were irradiated with electrons of $8 \mathrm{MeV}$ energy to a dose of about $10^{20}$ electrons $/ \mathrm{cm}^{2}$. Under these approximate starting conditions roughly $1 \mathrm{NV}$ defect is created per 100 vacancies, giving about $0.16 \mathrm{NV}$ per $10 \mathrm{~nm}$ diameter nanocrystal. The smallest size of NDs containing NV centres is reported to be $5 \mathrm{~nm}[34,35]$ in DNDs. DND powder was irradiated with $2.5 \mathrm{MeV}$ protons in a linear accelerator with a dose of $5 \times 10^{15} \mathrm{~cm}^{2}$ to demonstrate fluorescence emission. Finally, nonirradiated DNDs have been isolated and NV has been observed, showing for the first time a blinking behaviour [36].

\section{Coherent control of single spins}

NV centre spin shows a polarization under optical illumination. Over the years, it has been determined that optical excitation causes the ground $m_{s}=0$ state to become occupied and fully polarized $[12,37]$. The models for spin polarization are based on the existence of a singlet electronic state whose energy level lies between the ground and the excited state triplets. Transitions into this singlet state occur primarily from $\mathrm{m}_{\mathrm{s}}= \pm 1$ states, whereas decay from the singlet leads primarily to the $m_{s}=0$ ground state. If the remaining optical transitions are spin-preserving, this mechanism should fully polarize the NV centre into the $m_{s}=0$ ground state (Figure 3). Studies of coherent population trapping and Stark shifts indicate that local strain fields in diamond can mix the excited state spin projections, leading to optical transitions that do not preserve spin. On the whole, a picture of the NV centre is emerging in which some of the optical transitions preserve spin, while (depending on the degree of strain in the crystal) others do not. Most current research on the NV centre in diamond relies on optical detection of its ground state spin. Experimentally, an NV centre prepared in the $\mathrm{m}_{s}=0$ state fluoresces more strongly than an $\mathrm{NV}$ centre prepared in the $\mathrm{m}_{s}=1$ state. At room temperature, this allows for effcient detection of the average spin population. The same mechanism which leads to optical spin polarization provides the means to optically detect the spin state. Non-resonant excitation (typically at $532 \mathrm{~nm}$ ) excites both the $\mathrm{m}_{s}=0$ and $\mathrm{m}_{s}= \pm 1$ optical transitions. However, because the intersystem crossing occurs primarily from the $m_{s}= \pm 1$ excited state, population in $m_{s}= \pm 1$ ground state undergoes fewer fluorescence cycles before shelving in the singlet state. The $m_{s}= \pm 1$ 

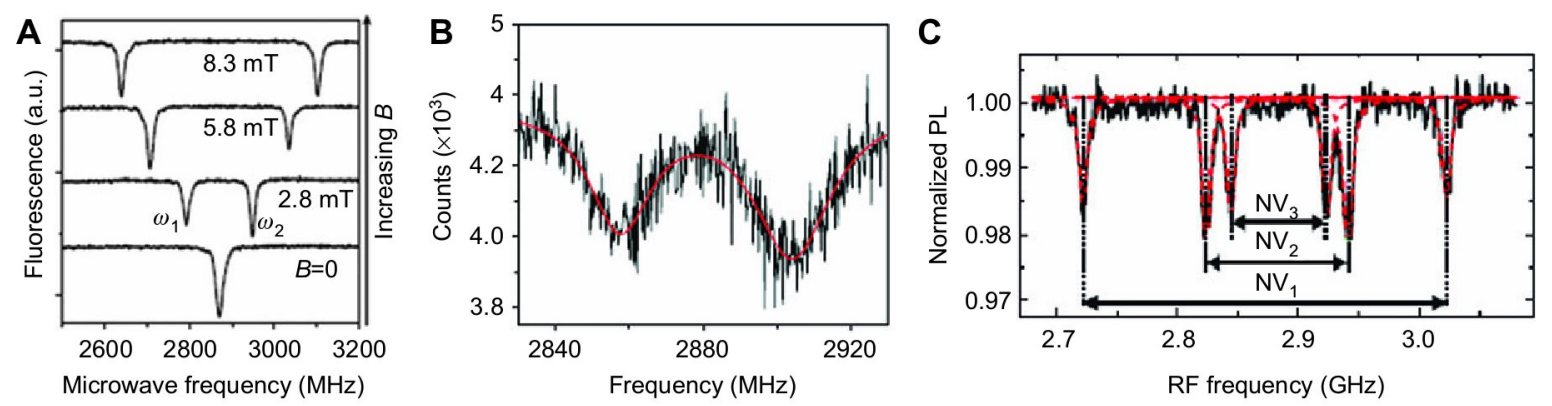

Figure 3 (A) Optically detected magnetic resonance spectra for a single NV defect at increasing magnetic field (from bottom to top) in a nanodiamond [6]. Reprinted by permission from Macmillan Publishers Ltd: Nature (http://www.nature.com/nature/journal/v455/n7213/abs/ nature07278.html), copyright (2008) (B) ODMR spectra measured on a single NV centre found in a DNDs [36], showing a lift of degeneracy due to strain. Reprinted by permission from Macmillan Publishers Ltd: [Nature Nanotechnology] (http://www.nature.com/nnano/journal/v5/ n5/abs/nnano.2010.56.html), copyright (2010). (C) Ensemble optically detected ESR on a (110) bulk type Ib diamond, showing 3 orientations of the NV axis with respect to the applied $B$ field direction. The fourth orientation is not visible due to specific crystallographic axis orientation of the diamond. Reprinted with permission from B. J. Maertz, A. P. Wijnheijmer, G. D. Fuchs, M. E. Nowakowski, and D. D. Awschalom, Appl. Phys. Lett. 96, 092504 (2010), Copyright 2010, American Institute of Physics.

states thus fluoresce less than the $m_{s}=0$ state, with a difference in initial fluorescence of $20 \div 40 \%$. It is worth noting that the $20 \div 40 \%$ contrast in room-temperature optically detected magnetic resonance (ODMR) experiments arises from a combination of imperfect polarization and imperfect readout. ODMR for $\mathrm{NV}$ is typically performed at the single defect level, while an equivalent optically detected electron spin resonance can be also applied at ensemble level. Both methods can be in continuous-wave $(\mathrm{CW})$ and pulsed operation of the microwave signal. For single defect measurement a confocal microscope is used and single particles with single photon emission are identified prior of the microwave excitation [38]. In ensemble measurement the excitation of the diamonds is done using a wide-field optical microscope. For CW measurements, microwave and optical excitation is applied at constant power to the NV centre, and the fluorescence intensity into the phonon sideband is measured as a function of the microwave frequency. The continuous $532 \mathrm{~nm}$ excitation polarizes the electron spin into the brighter $\mathrm{m}_{\mathrm{s}}=0$ state; when the microwave frequency is resonant with one of the spin transitions $\mathrm{m}_{s}=0, \pm 1$, the population is redistributed between the two levels, and the fluorescence level decreases. In the absence of an applied magnetic field, the electron spin resonance signal occurs at $2.87 \mathrm{GHz}$ (zero magnetic field splitting). When an external magnetic field is applied to the $\mathrm{NV}^{-}$centre, it does not affect the $\mathrm{m}_{s}=0$ states nor the ${ }^{1} \mathrm{~A}$ state (because it has $\mathrm{S}=0$ ), but it splits the $\mathrm{m}_{s}= \pm 1$ levels, the two frequency transitions are shifted apart. In pulsed operation specific temporal sequence of optical and microwave excitations are established as described in the next paragraph. If a magnetic field is oriented along the defect axis and reaches about $1027 \mathrm{G}$ (or $508 \mathrm{G}$ ) then the $\mathrm{m}_{s}=-1$ and $\mathrm{m}_{\mathrm{s}}=0$ states in the ground (or excited) state become equal in energy; they strongly interact resulting in so-called spin polarization, which strongly affects the intensity of optical absorption and luminescence transitions involving those states.

In Figure 3 we show typical ODMR of NV centres in bulk diamond, NDs, DNDs at the single centre level and optically detected ESR in ensemble.
There is an additional splitting of the $m_{s}= \pm 1$ energy levels, which originates from the "hyperfine" interaction between the nuclear and electron spins. In this case it is mostly associated to coupling to ${ }^{14} \mathrm{~N}$ or ${ }^{13} \mathrm{C}$ nearby nuclear spin [39]. Thus finally, the optical absorption and luminescence from the $\mathrm{NV}^{-}$centres consists of roughly a dozen sharp lines with a separation in the $\mathrm{MHz}-\mathrm{GHz}$ range, and all those lines can be resolved, given proper sample preparation.

The typical pulse sequence that is used to control NV spin properties is based on the measurement of Rabi nutations, corresponding to population oscillations between the ground $\mathrm{m}_{s}=0$ and excited $\mathrm{m}_{s}=1$ states in a small applied magnetic field [4(A)]. In fact the $\mathrm{m}_{s}=0$ to $\mathrm{m}_{s}=1$ spin transition of the NV centre constitutes an effective two-level system. This transition is driven with a resonant microwave excitation after initial polarization of the population in the $\mathrm{m}_{s}=0$ sublevel. The resonant microwave pulse is of varying duration $t$ and the frequency is initially calibrated using an ODMR measurement in the same $B$ field. The population remaining in $\mathrm{m}_{\mathrm{s}}=0$ is measured read out time, applying again an excitation green pulse. The result is a typical Rabi oscillation, whose frequency depends on the microwave power. For a given microwave power, Rabi nutations are used to calibrate the pulse length required to flip the spin from $\mathrm{m}_{s}=0$ to $\mathrm{m}_{s}=1$; this is known as a $\pi$ pulse, because it corresponds to half of the Rabi period. A $\pi / 2$ pulse brings the centre in a superposition of $m_{s}=0.1 \mathrm{spin}$ states. Rabi nutations corresponds to driven spin dynamics. We can observe the free dynamics, by generating a superposition of spin and letting them evolve freely and then rephasing them. The sequence is $\pi / 2, t, \pi / 2 . \pi / 2$ pulse is calibrated from the Rabi nutation. The spin population follow a decay on timescale of $\mathrm{T}_{2}{ }^{*}$, known as electron spin dephasing time. The dephasing time is the timescale on which the two spin states $\mathrm{m}_{s}=0$ and $\mathrm{m}_{s}=1$ accumulate random phase shifts relative to one another. For the NV centre, these random phase shifts arise primarily from the effective magnetic field created by a complicated but slowly-varying nuclear spin environment. To understand this environment in greater detail, it is necessary 
to employ more complicated pulse sequences which eliminate the phase shifts associated with the static ${ }^{14} \mathrm{~N}$ spin and quasi-static effects of the environment. This is addressed by a spin-echo sequence (Figure 4B). These frequency shifts can be eliminated by using a spin-echo (or Hahn echo) technique. It consists of the sequence $\pi / 2-\tau-\pi-\tau-\pi / 2$ (Figure 4B), where $\tau$ are durations of free precession intervals [37, 40]. The Hahn sequence begins by preparing a superposition of electron spin states. This superposition precesses freely for a time $\tau_{1}$, so that, for example, the $\mid 1>$ component picks up a phase shift relative to the $\mid 0>$ component. The $\pi$ pulse in the middle of the spin-echo sequence flips the spin. Assuming that the environment has not changed since the first free precession interval, the $\mid 1>$ component will pick up another phase during the second free precession interval. When the two wait times are precisely equal, $\tau_{1}=\tau_{2}$, the random phase shift factors out, so the final $\pi / 2$ pulse puts all of the population back into $\mid 0>$. When the wait times are unequal, the Hahn sequence behaves like a Ramsey sequence. Figure 4B shows a series of spinecho peaks and the significance of the coherence time of the spin. The longest coherence time of $1.8 \mathrm{~ms}$ ever achieved in solid state corresponds to NV centre spin [41], recently measured in ultra-pure or electronic grade ( $\mathrm{ppb} \mathrm{N}$ concentration) ${ }^{12} \mathrm{C}$ enriched bulk diamond. Long coherence times are particularly relevant in the application of NV as a magnetic sensor. Recently an outstanding application of these methods in living cell has been achieved, opening the possibility to measure small magnetic field effects in life science and specifically in neuroscience [42].

\section{Optical properties}

This section reviews the optical properties of NV centre in diamond including photochromism, two-photon absorption and nonlinearities.
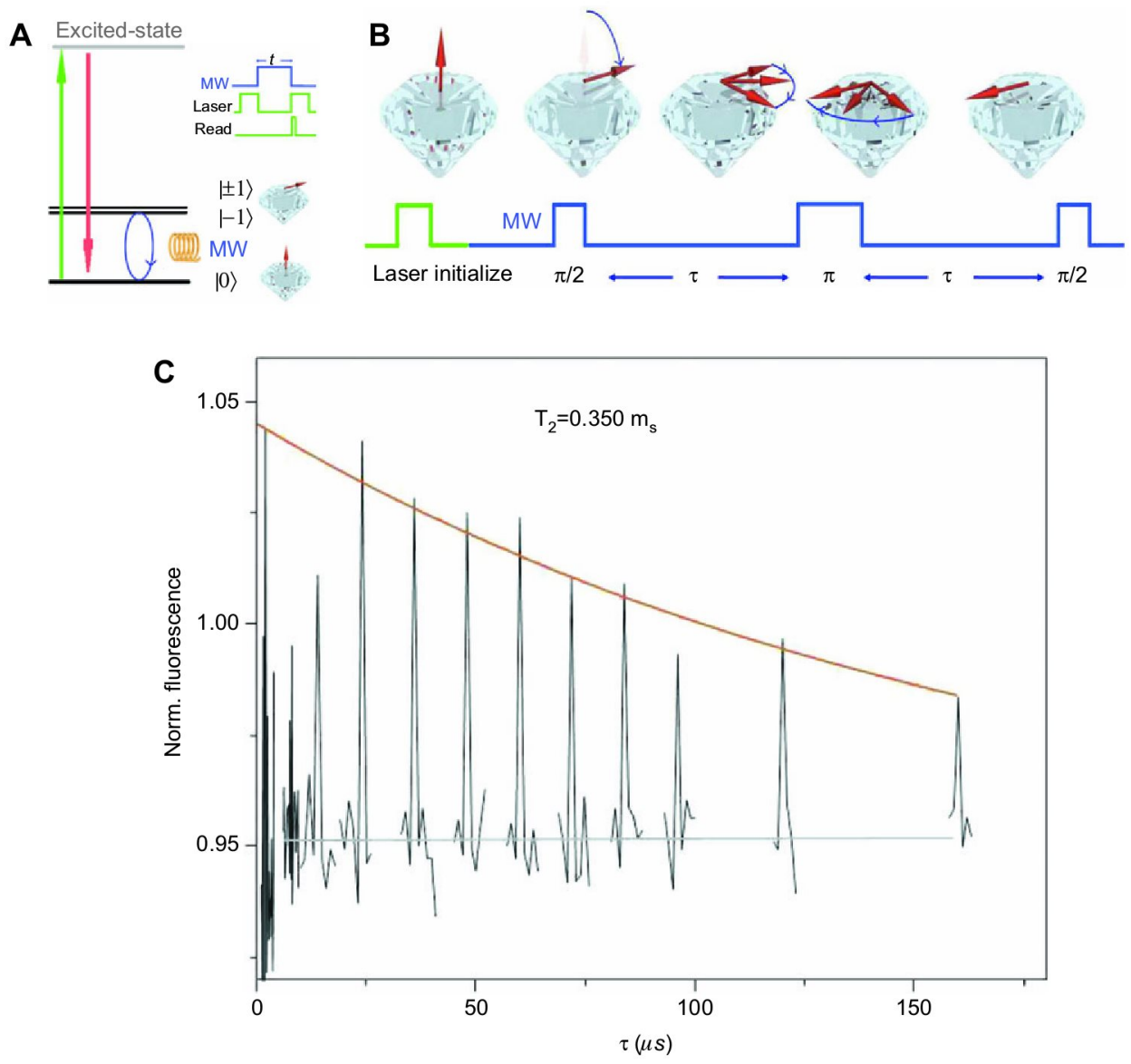

Figure 4 Illustration of typical microwave sequences described in Sec. 4 used for coherent control of single NV spins. Specifically (A) Rabi oscillation, where a resonant microwave pulse causes population oscillations between the ground state sublevels $\mathrm{m}_{s}=0$ and $\mathrm{m}_{s}=1$. (B) Typical spin-echo sequence for refocusing of spins by a pulse of resonant microwave. (C) Hahn echo decay curve of a single spin of NV centre with ${ }^{14} N$ nitrogen isotope recorded at room temperature. During each echo sequence, the first time $\tau_{1}$ was fixed and $\tau$ indicates the second interval that was varied. The peak height decays on the timescale called coherence time. Decoherence leads to a reduction of the echo amplitude as the pulse interval is increased. The orange curve is an exponential decay fit indicating a phase memory time of $350 \mu s$. If the environment were completely static, the spin-echo signal would be unity regardless: a non-trivial spin-echo signal means that the environment must be evolving on a timescale faster than the coeherence time. Reprinted by permission from Macmillan Publishers Ltd: Nature Physics (http://www.nature. com/nphys/journal/v2/n6/abs/nphys318.html), copyright (2006). 


\subsection{Photochromism}

Depending on the surrounding impurities which serve as electron donors or acceptors, the $\mathrm{NV}$ defect centre occurs in two charged states: neutrally charged $\mathrm{NV}^{0}$ and negatively charged $\mathrm{NV}^{-}$, with zero phonon lines at $575 \mathrm{~nm}(2.156 \mathrm{eV})$ and 637 $\mathrm{nm}(1.945 \mathrm{eV})$, respectively. It is well known that the energy level of $\mathrm{NV}^{-}$consists of a spin triplet ground state ${ }^{3} \mathrm{~A}$, a triplet excited state ${ }^{3} \mathrm{E}$ and a metastable spin singlet state ${ }^{1} \mathrm{~A}$. In contrast, a two level system with absence of a metastable state is generally accepted in $\mathrm{NV}^{0}$. The two charged states can be determined by their emission spectra, as illustrated in Figure 1B. In general, these two charge states can co-exist but a single charge state often dominates [43]. At different excitation conditions, the two charged states can transform from one to the other, known as photochromic effect and observed in both bulk diamond and nanocrystals [21, 44-46].

The proportion of $\mathrm{NV}-\mathrm{NV}^{0}$ was observed as a function of the irradiation of intensity and laser wavelength [46]. Under intense photon irradiation, the $\mathrm{NV}^{-}$can be photoionized to create the neutral charge state or vice versa. Reversible photochromic effect has been observed in NV centre ensemble embedded in bulk diamond prepared both by CVD and HPHT methods [47]. Under intense excitation by a laser beam at the wavelength of $532 \mathrm{~nm}$, the $\mathrm{NV}^{0}$ emission decreases and even disappears. The photochromism was reversible when reducing the excitation intensity [47]. Reversible transforming the $\mathrm{NV}^{0}-\mathrm{NV}^{-}$in a single $\mathrm{NV}$ centre embedded in bulk diamond has also been observed when increasing the intensity of the excitation power at the wavelength of $514 \mathrm{~nm}$ [45]. These observations were attributed to the ambient environment which affects the electron excitation and capture dynamics.

In contrast to bulk diamond, irreversible transform of a single $\mathrm{NV}^{-}$color centre to a single $\mathrm{NV}^{0}$ centre in nanodiamond with average size of $20 \mathrm{~nm}$ has been observed excited by a CW laser [47]. Once the excitation power at the wavelength of $532 \mathrm{~nm}$ exceeds $10 \mathrm{~mW}$, stable $\mathrm{NV}^{0}$ centre was created without any relaxation after $20 \mathrm{~h}$. Similar observation of irreversible creation of $\mathrm{NV}^{0}$ centre in nanodiamond has been reported using a pulsed laser excitation at the peak intensity of $50 \mathrm{GW} / \mathrm{cm}^{2}$ [44].

\subsection{Two-photon absorption}

The NV defect centre is known as trigonal symmetry $\left(\mathrm{C}_{3 \mathrm{v}}\right)$ and there is no centre of symmetry, therefore the selection rule of single photon allowed optical transition will not forbid the two-photon absorption. Two-photon induced fluorescence in NV centre embedded in Type Ib diamond has been observed by a pulsed laser at the wavelength of $1064 \mathrm{~nm}$ and the pulse width of 7.5 ps [48]. The two-photon induced emission spectrum was essentially identical to that excited by single-photon absorption, except that relative more intense emission from $\mathrm{NV}^{0}$ was detected. The two-photon absorption cross section has been identified as $(0.45 \pm 9.23) 10^{-50} \mathrm{~cm}^{2} \cdot \mathrm{s} /$ photon, nearly 30 times weaker than that of rhodamine B. A theoretical study of the two-photon absorption cross section of NV centre in diamond via configuration interaction with single excitation and complete active space self-consistent field methods showed a good agreement with the measurements [49].

\subsection{Nonlinearities}

Diamond has been identified as a good candidate for optoelectronic devices owing to its unique optical and spin properties. In spite of pure diamond has a centre for symmetry, second harmonic generation has been observed in ND, effect attributed to the symmetry perturbation at the surface [50]. Strong third harmonic generation has also been observed in NDs with average size of $100 \mathrm{~nm}$ which is far smaller than the coherent length [50]. The real part of the third-order nonlinear susceptibility has been determined as $\chi^{(3)}=0.4 \times 10^{-13} \mathrm{esu}$ at the wavelength of $1054 \mathrm{~nm}$. The optical Kerr nonlinearity has also been observed in a Z-scan measurement with a two orders of magnitude higher value $\chi^{(3)}=-3 \times 10^{-11}$ esu at the wavelength of $580 \mathrm{~nm}$ [50]. The optical nonlinearity of NV centre in bulk diamond has also been observed. The thirdorder nonlinear index in diamond has been measured using a two-beam coupling setup with $\operatorname{Re}\left(\chi^{(3)}\right)=(-1.7 \pm 0.4) \times 10^{-6}$ $\mathrm{cm}^{2} / \mathrm{W}$ and $\operatorname{Im}\left(\chi^{(3)}\right)=(1.5 \pm 0.6) \times 10^{-6} \mathrm{~cm}^{2} / \mathrm{W}$ [51].

\section{Magnetic sensor at the nanoscale and magneto-optical imaging}

The recent past has seen major advances in imaging magnetic structures at the nano- or even single atom level. Under low temperature and ultrahigh vacuum conditions spin polarized tunneling as well as exchange force microscopy have yielded unprecedented insight into surface bound magnetic structures. Under ambient conditions magnetic resonance imaging (MRI) is the canonical method especially in biological science. Although millimetre resolution MRI is a well established clinical technique it is only recently that the sensitivity has been improved enough to image single cells. Low temperature magnetic resonance force microscopy has succeeded in detecting single electrons and small nuclear spin ensembles. In contrast to optical systems, in magnetic resonance imaging the spatial resolution is not determined by diffraction; rather, it is limited by magnetic field sensitivity, and in principle it is possible to achieve a resolution much better than the optical wavelength used. NV-based magnetic field measurements rely on the property that single-electron spin states can be optically detected at room temperature. Hence by combining magneto-optical spin detection enabled by NV in diamond with scanning technology (Atomic Force Microscopy, AFM), nanometer-scale magnetic objects and possibly even single spins can be imaged under ambient conditions [52].

Two approaches have been used to sense magnetic fields using diamond and to image the magnitude and the vector magnetic field.

One is based on CW operation and single emitter detection from NDs; in this case a magnetic cantilever tip is used to produce a magnetic gradient, which is sensed by ODMR measurements of NV centres [6]. A commercial AFM was integrated with an optical confocal microscope scanning 
stage. The confocal image in the presence of the cantilever and microwave excitation constitutes a $2 \mathrm{D}$ image of the gradient magnetic field and allows to locate the NV spin with nanometric resolution. By attaching directly the nanodiamond to the cantilever tip NV centres have been used as scanning probe magnetometer, thus obtaining a super-resolved magneto-optical image of a nickel magnetic nanostructure prepared by electron beam lithography (Figure 5).

The second approach [5], shows the use of single NV centres in bulk material to measure dynamic (AC) magnetic fields with a sensitivity of $30 \mathrm{nT} / \sqrt{\mathrm{Hz}}$, limited only by the 600 $\mu$ s decoherence time due to the presence of ${ }^{13} \mathrm{C}$ spin bath. In this case the varying magnetic field is measured using a spin echo sequence, the observed peak of the spin-echo signal varies periodically as the amplitude of the external AC field is increased. Since the spin-echo signal is a measurement of the probability of the NV spin to be in $\mathrm{m}_{s}=0$, in the presence of a varying magnetic field a phase shift depending on the field is accumulated compared to the typical envelope measured in absence of the AC magnetic field. A static (DC) magnetic field is used to increase the resolution of the measurement. The minimal field detectable is given by $\delta B \approx \frac{\hbar}{g \mu_{B} \sqrt{T_{2} T}}, T_{2}$ is the electronic spin coherence time, $T$ is the measurement time, $\mu_{B}$ is the Bohr magneton, $\hbar$ is Planck's constant divided by $2 \pi$, and $g \approx 2$ is the electronic Lande g-factor. With $\mathrm{T}_{2}$ from 0.1 to $1 \mathrm{~ms}$ sensitivity of few $\mathrm{nT} / \sqrt{\mathrm{Hz}}$ can be achieved. At a distance of $10 \mathrm{~nm}$, the spin of a single electron produces a magnetic field of about $1 \mathrm{mT}$, and the corresponding field from a single proton is a few nT. A sensor based on NV able to detect such magnetic fields with nanometre spatial resolution would enable powerful applications, ranging from the detection of magnetic resonance signals from individual electron or nuclear spins in complex biological molecules, to readout of classical or quantum bits of information encoded in an electron or nuclear spin memory.

A key feature of NV sensors is that it can be localized within a region of about $10 \mathrm{~nm}$, either in direct proximity to a diamond surface or within a nanodiamond. The present limitation is, however, due to a typically shorter coherence time of NV in commercial NDs attributed to other spin impurities, that limits the magnetometer resolution to $0.5 \mu \mathrm{T} / \sqrt{\mathrm{Hz}}$. Efforts in producing NDs which could approximate the best sensitivity obtained in bulk material of $4 \mathrm{nT} / \sqrt{\mathrm{Hz}}$ is an ongoing research endeavor. Methods to increase the coherence time by refocusing the spin using multiple sequences are also under investigation. By using an appropriate pulse sequence bath-related magnetic noise can be completely refocused, effectively allowing to extend the free evolution time up to the limit corresponding to the imposed by the spin-lattice relaxation time of the order of $\approx 10 \mathrm{~ms}$ [53], depending on the type of diamond.

An alternative approach for NV-based magnetometry has been proposed [7] and recently verified [54-56] employing high density NV centres (mostly in type Ib bulk diamond) also referred to as ensemble magnetometry. A principle advantage of this approach over other spin precession magnetometers is the high achievable density $n$ of sensing spins. This improves the sensitivity to fields that are homogeneous over the magnetometer volume, because the projection noise per unit volume decreases as $1 / \sqrt{n}$. NV centres can be created in controlled densities by implanting high-purity diamond with nitrogen ions and subsequently annealing the sample to recombine

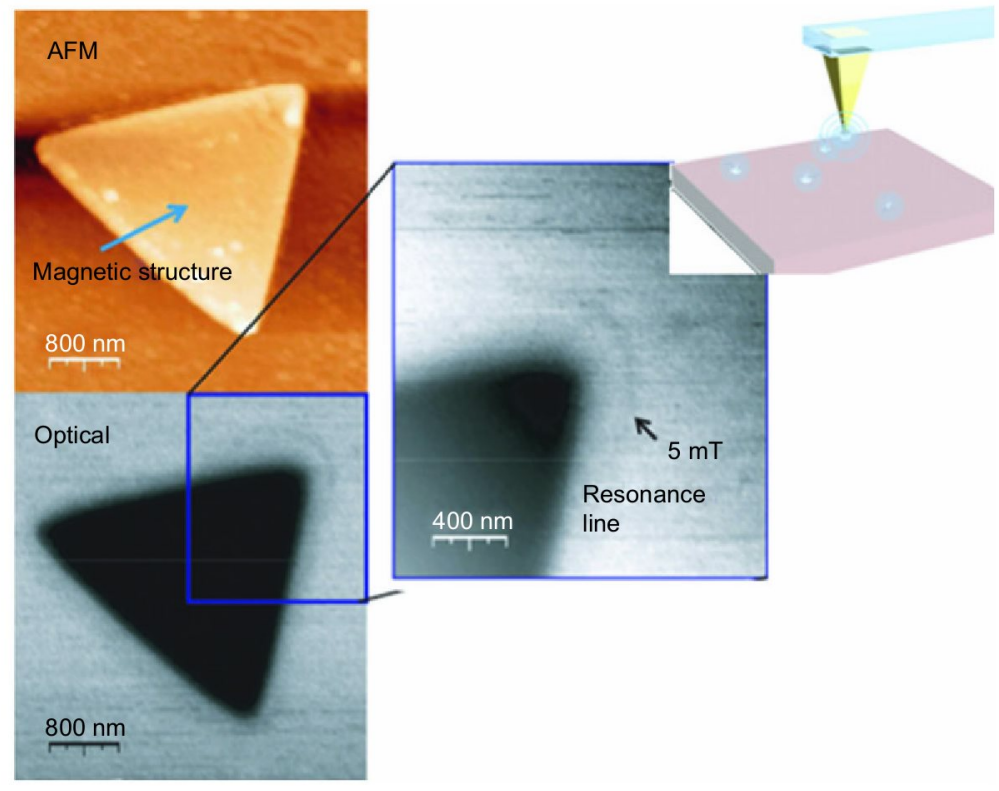

Figure 5 Relative magnetic field reconstruction using a scanning probe single spin magnetometer. AFM image of a nickel magnetic nanostructure prepared by electron beam lithography and a magneto-optical image of the same structure, recorded using a single nitrogen-vacancy centre on the AFM tip as light source and magnetometer. The magnetic field resolution is given by the width of the dark lines, which are about $20 \mathrm{~nm}$. Reprinted by permission from Macmillan Publishers Ltd: Nature (http://www.nature.com/nature/journal/v455/n7213/full/nature07278. html), copyright (2008). 
the nitrogen with vacancies. The detection system is similar but using a wide-field optical microscope and CCD camera. In particular Lai and co-workers [57] used a HPHT singlecrystal diamond with NV centre created from the initially embedded nitrogen impurities, by irradiation with a highenergy electron beam and annealing for $2 \mathrm{~h}$ at $850^{\circ} \mathrm{C}$. With the applied irradiation dose of $10^{13}$ electrons $/ \mathrm{cm}^{2}$, a high density of about $200 \mathrm{NV}$ centres $/ \mathrm{cm}^{3}$ can be created. Maertz and coworkers [54] for vectorial magnetic field measurement used untreated type Ib diamonds. At high spin densities, NV paramagnetic impurities and NV/NV interactions may limit the sensitivity of the magnetometer. In particular, substitutional nitrogen impurities with one bound electron become a sizable source of dephasing in high-density samples. Diamonds with a higher concentration of $\mathrm{NV}$ centre will produce a stronger fluorescence signal, but in general also contain a higher concentration of nitrogen paramagnetic impurities, which further limits the coherence time. A recent result shows an NV ensemble magnetic field imager used to measure AC magnetic field pattern with sub-micron resolution over a $140 \mu \mathrm{m} \times$ $140 \mu \mathrm{m}$ field of view, giving single pixel sensitivity of 100 $\mathrm{nT} / \sqrt{\mathrm{Hz}}$ [58]. The NV sensors were confined to a thin layer on the diamond surface and placed within a sub-micron distance from the magnetic field source.

Using ensemble magnetometry a magneto-optical imaging of the vectorial magnetic field has been proven. In [54] optically detected ESR was used while in [58] the magnetic field measurements were improved using a pulse sequence. In both cases the spatial resolution can be significantly improved by implementing stimulated emission depletion microscopy, as it will be described in the next section. This improvement could bring the spatial resolution to a level comparable with magnetic force microscopy.

Another approach to increase the sensitivity of ensemble magnetometry is by reading out the NV spin state using infrared dark state absorption at $1042 \mathrm{~nm}$ [28]. Under continuous optical pumping, the population of NV centres in the metastable singlet is detected by monitoring the transmission of a $1042 \mathrm{~nm}$ probe beam and use this to read out the spin polarization of the ensemble. With this technique, measurement contrast and collection efficiency can approach unity, leading to an overall increase in magnetic sensitivity. In fact in addition to the spin coherence time, the low collection efficiency in detecting NV emission from bulk diamond together with the quantum noise associated with spin projection are limiting factor in magnetometry sensitivity. With this method, despite of a coherence time of $150 \mathrm{~ns}$ of the starting material due to the high concentration of $\mathrm{NV}\left(\approx 10^{17} / \mathrm{cm}^{3}\right)$, a sensitivity of $5 \mathrm{pT}$ in one second of acquisition and bandwidth from DC to a few megahertz has been achieved. The drawback of this method is the $77 \mathrm{~K}$ operation for observing the infrared absorption. The present technique can be extended to room temperature operation by employing a cavity with finesse 200 .

\section{Nanoscopy and sub-diffraction imaging}

Due to the diffraction of light, the resolution of conventional light microscopy is limited as stated by Ernst Abbe in 1873 .
A good approximation of the resolution attainable is the full width at half-maximum of the point spread function, and an optimized wide-field or confocal microscope with high numerical aperture and imaging by visible light usually reaches a resolution of $\sim 250 \mathrm{~nm}$. Super-resolution techniques allow the capture of images with a higher resolution than the diffraction limit, pushing fluorescence microscopy to the nanoscale and opening the field of nanoscopy, once the prerogative of electron and scanning probe microscopes. They fall into two broad categories, one captures information contained in evanescent waves, and the second uses experimental techniques and photo-physics effects in the material to obtain a superresolution image. The first category of sub-wavelength imaging techniques relying on the collection of evanescent waves includes those that utilize the Pendry superlens [59] and near field scanning optical microscopy; however, these methods are not so widespread in the applications yet, limited by the severe near field operation requirement. There are two major groups of methods that rely on the fundamental and quantum physics properties of the material known as deterministic and stochastic superresolution. Both methods rely on the fact that the fluorescent markers within the same diffraction volume can be resolved if they are not emitting at the same time. The major difference is that in the deterministic techniques the area in which the markers can signal is determined by the position of the foci. Specifically, deterministic methods are based on fluorophores showing a nonlinear response to excitation, these methods include Stimulated emission depletion microscopy (STED), ground state depletion (GSD) microscopy, REversible Saturable OpticaL Fluorescence Transitions (RESOLFT). Stochastical methods rely on the chemical complexity of many nanoemitters showing complex temporal behaviour, which can be used to make several close-by fluorophores emit light at separate times and thereby become resolvable in time.

In this section we focus on deterministic super-resolution imaging methods based on the extreme photo-stability of NV centres in monolithic diamond and its spin polarization. Consequently, the successful application of existing high resolution techniques on the NV centres has paved the way for many NV based applications which were not possible before. The demonstration of STED with NV centre opened new avenues both in terms of nanofabrication [32] of NV centres and in terms of spin manipulation without diffraction limitation. The first application of STED microscopy using albuminconjugated fluorescent NDs has been recently demonstrated [60].

\subsection{Stimulated emission depletion microscopy (STED)}

The concept of STED microscopy was first proposed and demonstrated in 1994 [61]. STED utilizes the process of stimulated emission to deplete the excited state (in NV ${ }^{3} \mathrm{E}$ ) from which the spontaneous fluorescence emission occurs between 650 and $750 \mathrm{~nm}$. This is possible if the stimulated emission rate is much larger than that of the spontaneous decay, which is clearly the case of NV due to its long spontaneous emission 
life time (11.7 $\mathrm{ns}$ in bulk and $25 \mathrm{~ns}$ in NDs). Moreover, the phononic coupling in the ${ }^{3} \mathrm{~A}$ state enables quenching of the excited NV centres by stimulated emission more efficiently $[1,11]$. A steep photoluminescence decay is observed when the STED beam with intensity $I_{\text {STED }}$ is applied to NV, yielding a saturation intensity of $I_{S}=6.6 \mathrm{MWcm}^{-2}$, while typical dye fluorescent beads have a $I_{S}=11 \mathrm{MWcm}^{-2}$ [62]. Since the fluorescence is completely switched off when $I_{\mathrm{STED}}>>3 I_{S}$ and the resolution increases with the ratio between the maximum STED beam intensity and the saturation intensity, clearly diamond provides the better property for the maximum resolution achievable.

The STED technique uses two synchronized overlapping laser beams which fall consecutively on the sample, of which the first one (excitation laser) excites the fluorescent emitters in the sample to the excited state and the second (STED beam) depletes the excitation states. Stimulated emission happens from the region having both laser beams while spontaneous emission occurs in the region having only the first excitation beam. By shaping the STED beam as a doughnut beam, while the excitation beam is Gaussian, the spontaneous emission occurs only in the region where the STED beam is absent, defined by the centre of the doughnut-shaped beam, allowing to achieve a sub-diffraction emission area. This super-resolution recording is governed by the spatial overlapping of the photo-excitation and photoinhibition patterns, rather than the diffraction limitation. STED microscopy has demonstrated a resolution of 16-80 $\mathrm{nm}$ before being applied to NV in bulk diamond [1], where a world record resolution has been achieved of $8 \mathrm{~nm}$. This is mainly attributed to extreme photostability of NV compared to typical dye, allowing to push the STED beam intensity at $3.7 \mathrm{GW} / \mathrm{cm}^{2}$. In addition, NV possesses a high quantum efficiency (inferred to be 0.7 ) that contributes to a high cross section of stimulated emission, and also a broad emission, which allows to use a quite wide separation between excitation beam at $532 \mathrm{~nm}$ and STED at $775 \mathrm{~nm}$. The first STED experiment [1] has been demonstrated using type IIa bulk diamond untreated. The STED beam and the excitation beam were both pulsed lasers allowing for the best achievable resolution. For implementation of STED in NDs [3], the material used was from treated NDs following Chang and co-workers procedures [63]. A high fluence $40 \mathrm{keV} \mathrm{He} \mathrm{He}^{+}$ion beam is used to generate vacancies in type Ib, high-pressure high-temperature (HPHT) NDs. NDs as small as $25 \mathrm{~nm}$ have been produced and each particle can contain up to seven NV centres. In this case high repetition rate pulsed and CW lasers were used and 3D super-resolution has been achieved (Figure 6).
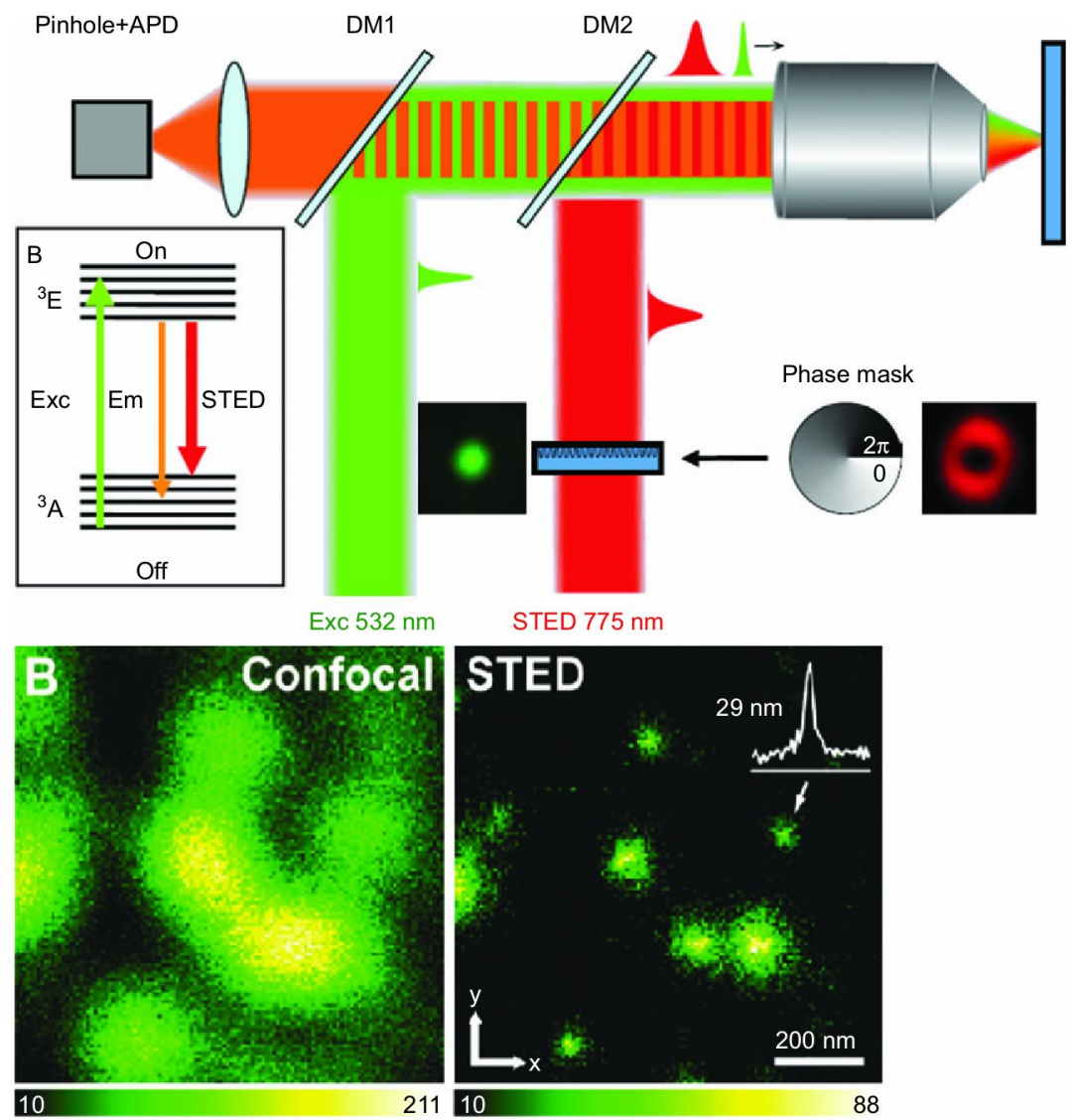

Figure 6 Typical STED scheme to image NV in diamond. Reprinted by permission from John Wiley and Sons: Small (http://onlinelibrary. wiley.com/doi/10.1002/smll.201000902/abstract), copyright (2010). At the bottom CW STED images of NDs containing NV centres, clearly resolved in the STED (right) but blurred in the confocal recording (left). Reprinted with permission from Kyu Young Han et al., ThreeDimensional Stimulated Emission Depletion Microscopy of Nitrogen-Vacancy Centers in Diamond Using Continuous-Wave Light, Nano Lett., 2009, 9 (9), 3323, Copyright (2009) American Chemical Society. 


\subsection{Ground state depletion microscopy involving excited-state and dark-state}

In Figure 7 a schematic of various type of deterministic superresolution imaging with $\mathrm{NV}$ centres are shown, to clarify the distinction between the different methods [64]. In the case of ground state depletion (GSD) to ${ }^{3} \mathrm{E}$, the ground state $\left({ }^{3} \mathrm{~A}\right)$ is depleted and the emitter is shelved in the ${ }^{3} \mathrm{E}$. In this case a doughnut $532 \mathrm{~nm}$ beam is used to shelve the centre in its excited state, meaning that the emission occurs in all the sample, and the localisation below the diffraction limit corresponds to a dark point [2]. Post-processing algorithms for mathematical deconvolution with the calculated point spread function are used to obtain the positive image. Alternatively, the positive image can also be obtained by employing a second, periodically modulated excitation beam in combination with difference imaging, using a lock-in amplifier, thus not suffering of deconvolution artifact. Concerning the preparation of the states, GSD via saturation of the fluorescent state behaves like STED. The main disadvantage is the achievable signal to noise ratio: the contrast relies on the fact that the emitter under observation does not contribute to the recorded signal since it is not excited while coinciding spatially with the doughnut centre. All the other emitters in the same area of diffraction, fluoresce and thus lead to a high fluorescence background. For dense samples, the number of counts becomes so high that sensitive single photon detectors easily saturate.

Therefore less sensitive detectors have to be used. However, the method is quite simple and several groups implemented to obtain diffraction unlimited NV imaging. With this method resolution of $8 \mathrm{~nm}$ in a low-density NV sample of a type IIa bulk diamond has been obtained. A variant of GSD has been achieved via the metastable state or dark state of NV, which uses a recently discovered physical effect [4]. The dark state is emptied by blue light via transitions to higher states, while the red light may excite the $\mathrm{NV}^{-}$centre to ${ }^{3} \mathrm{E}$, and it also efficiently transfers the $\mathrm{NV}^{-}$to a metastable dark state, thereby depleting its ground state. Excitation to ${ }^{3} \mathrm{E}$ is comparatively inefficient for blue light $<490 \mathrm{~nm}$, but this wavelength effiectively depopulates the dark state. Therefore, only the simultaneous irradiation by blue and red light yields strong luminescence. By using a pulse sequence of red $(638 \mathrm{~nm})$ and a blue $(473$ $\mathrm{nm}$ ) excitation sources a shelving and deshelving of the metastable state is achieved, respectively, switching on and off the NV spontaneous emission [4]. The dark state population increases with increased power of the blue shifted excitation beam. By a repeated pump-probe cycle of 473,638 , and 592 $\mathrm{nm}$ using photon correlation for long delay times, the lifetime of the dark state for a single $\mathrm{NV}^{-}$centre has been determined to be $150 \mathrm{~s}$. The GSD via dark state method therefore consists of an initial Gaussian beam to deshelve the metastable state and a doughnut beam that switches off some NV centres. A green read-out beam is used. This method has been established in type IIa diamond with a resolution comparable to other methods, but with a much lower power employed. One limitation of this method could be the dependence on the properties of the metastable state that can vary from type IIa or type Ib bulk diamond or various nanodiamonds. However, future avenues could be to exploit this process in conjunction with magnetic imaging or spin manipulation at the nanoscale.

\subsection{Reversible saturable optical fluorescence transitions based on NV ground state spin}

STED, GSD and dark state depletion are belonging to a wider category of super-resolution methods known as Reversible Saturable Optical Fluorescence Transitions (RESOLFT), since the emitter is spatially and temporarily switched off by forcing it to a state in which they cannot emit photons upon illumination. Spin-Resolft imaging can be applied to NV centres [65], providing an improved spatial resolution by illuminating the sample with a doughnut-shaped optical beam just before optical spin readout. Spins positioned directly in the centre of the doughnut beam are not affected by it. To achieve superesolution in SPIN-RESOLFT an initial Gaussian pulsed green laser is used to polarize the $\mathrm{NV}$ spins in the $\mathrm{m}_{s}=0$ ground state, a microwave pulse brings the spin to the state $\mathrm{m}_{s}= \pm 1$, and a second green laser with a doughnut-shaped selectively manipulates the spin only in the central part of the beam since it polarizes again the spin in the external part of the beam, allowing subdiffraction spin manipulation of NV centres. The physical principle is to switch the system between $\mathrm{m}_{s}=0$ and $m_{s}= \pm 1$ state with only $30 \%$ contrast. The advantage is that it can be implemented using much less power than STED or GSD, which is important for biological samples and its resolution depends on product of the length of the doughnut beam and its power. Therefore similar resolution of STED with a power of $1 \mathrm{~W}$ can be achieved with power of $0.1 \mathrm{~mW}$ as far as the pulse duration is long enough. Due to the poor signal to noise ratio however, the same quality image requires
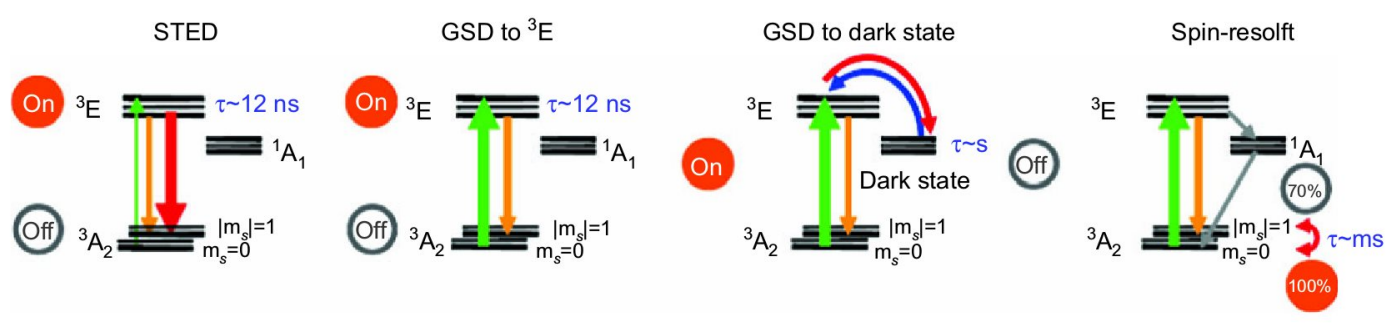

Figure 7 Summary and schematic of physical effects related to NV used to implement present deterministic sub-diffraction imaging. Reprinted with permission from D. Wildanger, J. R. Maze, and S. W. Hell Phys. Rev. Lett. 107, 017601 (2011) http://prl.aps.org/abstract/PRL/ v107/i1/e017601. Copyright (2011) by the American Physical Society. 
10,000 times longer time before being obtained, implying that eventually the total dose of light irradiation on the sample can be approximately of the same level as other methods. This can therefore impact in biological applications. A positive aspect of the method is that the beam pulse is only limited by the spin-state relaxation time $(>100 \mathrm{~ms})$, while STED pulse duration is limited by the excited state life time (11 ns). This method differentiates also from the others because it is not all-optical since a microwave pulse is required. This can induce a limitation since the spin has to be manipulated coherently and requires a pre-knowledge of the resonance frequency of each NV as well as of the coupling strength to the microwave. The method therefore can be challenging in the case of applications where NV centres are randomly oriented. Spin-Resolft imaging allowed to perform spatially selective Rabi oscillations and spin-echo measurements of two NVs apart of $150 \mathrm{~nm}$, unveiling stark differences owing to different local magnetic field environments. This method, enabling spatially selective spin-echo measurements for the two NV spins, revealed substantial differences in spin coherence times between close centres, indicating the presence of magnetic impurities in the local environment of one NV centre. The present challenge is to use STED or SPIN-RESOLFT to see dipolar coupling between $20 \mathrm{~nm}$ or less distant centres. For implementing SPIN-RESOLFT imaging methods, NV centres were created by irradiating the CVD diamond with $10 \% / \mathrm{cm}^{2}$ of nitrogen ions with an energy of $6 \mathrm{keV}$, corresponding to an estimated average implantation depth of $10 \mathrm{~nm}$. The sample was then annealed at $800^{\circ} \mathrm{C}$, yielding an observed $\mathrm{NV}$ concentration of approximately $0.6 \mathrm{NV} / \mu \mathrm{m}^{2}$.

\subsection{Super-resolution single spin manipulation and recording}

Recently STED microscopy has been employed to resolve NV spin states without diffraction limit [64]. This is based on adding a STED beam to a typical pulsed confocal-ODMR setup, consisting of a $532 \mathrm{~nm}$ polarization beam and a microwave pulse tuned to the ground state spin transitions in the presence of a static magnetic field. While the ODMR of the confocal revealed 4 resonance dips indicating the presence of at least $2 \mathrm{NV}$ centres in the diffraction limited confocal spot, by adding a STED beam $5 \mathrm{NVs}$ were clearly distinguished and each NV ODMR signal individually resolved. By changing the magnetic field the magnitude of the ODMR signal split changed dramatically, proving the ability to sense changes of external magnetic fields at the nanoscale. All conventional ODMR experiments such as high sensitivity AC and DC field sensing by echo sequences and relaxation times measurements can be performed at the same speed and noise level as in the confocal mode but without being limited by diffraction. For different spin interaction the effect of the STED beam on the spin state of NV has been considered testing it on Rabi oscillation and spin echo sequences. The result is that the STED beam preserves the spin state in Rabi oscillation, in other words protect the spin population from optical destruction, even improving the contrast. This is possibly due to the fact that stimulated emission occurs on $m_{s}= \pm 1$ states, and therefore correct possible polarization on the $\mathrm{m}_{s}=0$ state during the sequence. It has to be noted, however, that this spin preservation depends on the intensity of the STED beam, since the spin preservation occurs only when the dark-state is weakly populated. In the case of Hahn-echo sequences the effect of the STED beam induces a loss of information in the phase of the spin state.

\section{Conclusions and outlook}

Diamond NV centres are at the frontier of diffraction unlimited optical methods for spin manipulation and magnetic field sensing, clearly witnessing the development of outperforming novel technologies. In Figure 8, we highlight the here described experiments and future directions of the application of NDs defects. We have described the progress in material synthesis and in

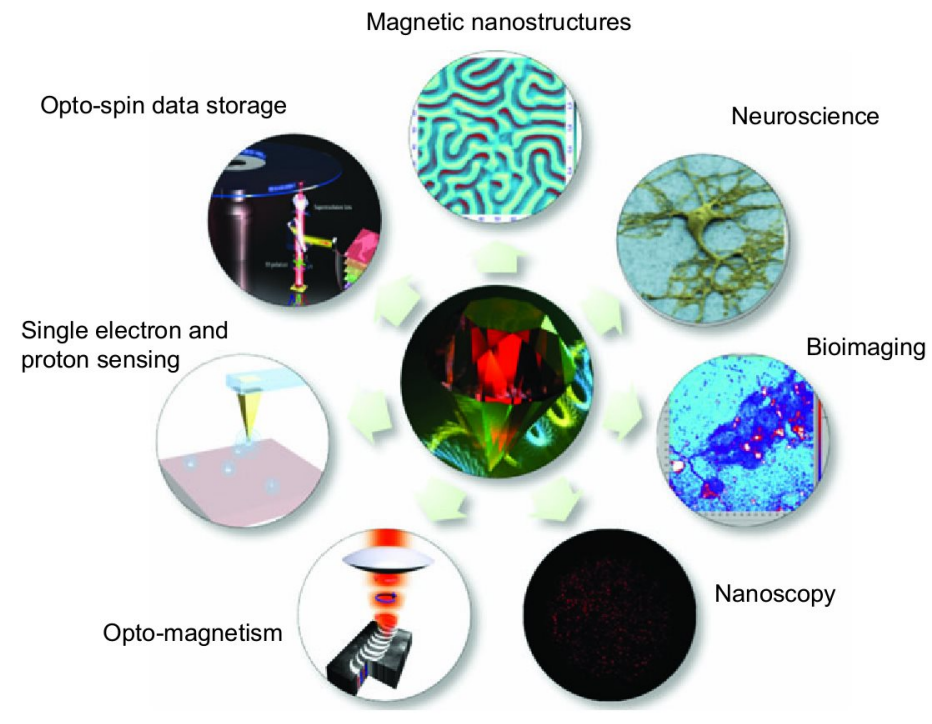

Figure 8 Summary of present and future applications of NV centres in diamond. 
the centre characterization in the last years which led to some groundbreaking results in several fields from quantum nanosensors to nanoscopy. However, further knowledge of its fundamental photo- and electrical-properties, have still to be unraveled, and some outstanding, though possible to surpass, technological challenges, are still a research endeavor, that will show even further important applications of these centres in the near future. We can perhaps imagine what scientists will be able to accomplish with the access to controllable and high quality nanodiamonds with NV centres. To emphasize the vast improvement in diamond material, in NV centres creation in both bulk and nanodiamonds, its increased availability and knowledge of its properties in various laboratories around the world, we mention how the super-resolution imaging technology has been enriched. Indeed, better material preparation and characterization via ODMR, Rabi nutation and spin-echo, allowed the improvement of existing techniques (such as STED and GSD), their further extension for sensing magnetic field at the nanoscale (STED-ODMR) or for nanofabrication of the centres. Moreover, new super-resolution methods have been established such as SPIN-RESOLFT. Owing to their superior photo-stability and spin polarization, a variety of super-resolution methods have been demonstrated when applied to NV centres in diamond. Among the reported super-resolution imaging methods based on NV centres, STED method shows its superiority over the others. A new world record of optical resolution down to $8 \mathrm{~nm}$ has been demonstrated using STED. The high signal noise ratio and the simplicity of this method without requirement of pre-knowledge of their orientation have enabled the widespread of STED in many NV based applications. Other super-resolution methods could benefit from more specific tailoring the fluorescence properties of $\mathrm{NV}$ or by exploiting functional physical effects as yet found or still to be determined. A natural future application in life science is also expected, where spin and magnetic sensing combined to super-resolution methods will be employed for further scientific discoveries. Other domains such as optical data storage that traditionally benefit from super-resolution methods and advances in nanophotonics, will benefit from $\mathrm{NV}$, by opening the possibility to bridge the gap between optical and magnetic data storage systems. In the domain of magnetic field sensing NV is becoming a star in quantum metrology, as a new addition to well established superconducting quantum interference devices, force sensors, Hall sensors, as well as Rb-based atomic sensors. Finally NV centres, even if not discussed here, occupies a special place as possible flying qubit for remote entangling of spin qubits in solid state, due to its optical-spin polarization properties and its possible integration in quantumhybrid-spin systems (e.g., NV and magnetic single molecules). Other deep centres as NV centre in diamond and in similar wide-bandgap semiconductors, such as $\mathrm{SiC}$ are under investigation $[18,66]$; we are witnessing either a new era of research of another NV-like centre in solid state or the establishing of novel advanced technologies in the above-mentioned fields.

\section{Acknowledgments}

The authors would like to acknowledge the financial support of the Australian Research Council Laureate Fellowship (FL100100099) scheme. SC is presently supported by the Australian Research Council under the Centre of Excellence for Engineered Quantum Systems.

\section{References}

[1] Rittweger E, Han KY, Irvine SE, Eggeling C, Hell SW. STED microscopy reveals crystal colour centres with nanometric resolution. Nat Phot 2009;3:144-7.

[2] Rittweger E, Wildanger D, Hell SW. Far-field fluorescence nanoscopy of diamond color centres by ground state depletion. Europhys Lett 2009;86:14001.

[3] Han KY, Willig KI, Rittweger E, Jelezko F, Eggeling C, Hell $\mathrm{SW}$. Three-dimensional stimulated emission depletion microscopy of nitrogen-vacancy centres in diamond using continuouswave light. Nano Lett 2009;9:3323-9.

[4] Han KY, Kim SK, Eggeling C, Hell SW. Metastable dark states enable ground state depletion microscopy of nitrogen vacancy centres in diamond with diffraction-unlimited resolution. Nano Lett 2010;10:3199-203.

[5] Maze JR, Stanwix PL, Hodges JS, Hong S, Taylor JM, Cappellaro P, Jiang L, Dutt MVG, Togan E, Zibrov AS, Yacoby A, Walsworth RL, Lukin MD. Nanoscale magnetic sensing with an individual electronic spin in diamond. Nature 2008;455:644-7.

[6] Balasubramanian G, Chan IY, Kolesov R, Al-Hmoud M, Tisler J, Shin C, Kim C, Wojcik A, Hemmer PR, Krueger A, Hanke T, Leitenstorfer A, Bratschitsch R, Jelezko F, Wrachtrup J. Nanoscale imaging magnetometry with diamond spins under ambient conditions. Nature 2008;455:648-52.

[7] Taylor JM, Cappellaro P, Childress L, Jiang L, Budker D, Hemmer PR, Yacoby A, Walsworth R, Lukin MD. Highsensitivity diamond magnetometer with nanoscale resolution. Nat Phys 2008;4:810-6.

[8] Neumann P, Beck J, Steiner M, Rempp F, Fedder H, Hemmer PR, Wrachtrup J, Jelezko F. Single-shot readout of a single nuclear spin. Science 2010;329:542-4.

[9] Buckley BB, Fuchs GD, Bassett LC, Awschalom DD. Spin-light coherence for single-spin measurement and control in diamond. Science 2010;330:1212-5.

[10] Neumann P, Kolesov R, Naydenov B, Beck J, Rempp F, Steiner M, Jacques V, Balasubramanian G, Markham ML, Twitchen DJ, Pezzagna S, Meijer J, Twamley J, Jelezko F, Wrachtrup J. Quantum register based on coupled electron spins in a roomtemperature solid. Nat Phys 2010;6:249-53.

[11] Davies G, Hamer M. Optical studies of $1.945 \mathrm{eV}$ vibronic band in diamond. P Roy Soc Lond A Mat 1976;348:285-98.

[12] Gruber A, Drabenstedt A, Tietz C, Fleury L, Wrachtrup J, von Borczyskowski C. Scanning confocal optical microscopy and magnetic resonance on single defect centres. Science 1997;276:2012-4.

[13] Togan E, Chu Y, Trifonov AS, Jiang L, Maze J, Childress L, Dutt MVG, Sorensen AS, Hemmer PR, Zibrov AS, Lukin MD. Quantum entanglement between an optical photon and a solidstate spin qubit. Nature 2010;466:730-4.

[14] Manson NB, Harrison JP, Sellars MJ. Nitrogen-vacancy centre in diamond: model of the electronic structure and associated dynamics. Phys Rev B 2006;74:104303.

[15] Batalov A, Jacques V, Kaiser F, Siyushev P, Neumann P, Rogers LJ, McMurtrie RL, Manson NB, Jelezko F, Wrachtrup J. Low temperature studies of the excited-state structure of negatively charged nitrogen-vacancy color centres in diamond. Phys Rev Lett 2009;102:195506. 
[16] Lenef A, Rand SC. Electronic structure of the NV centre in diamond: theory. Phys Rev B 1996;53:13441-55.

[17] Aharonovich I, Castelletto S, Simpson DA, Stacey A, McCallum J, Greentree AD, Prawer S. Two-level ultrabright single photon emission from diamond nanocrystals. Nano Lett 2009;9:3191-5.

[18] Weber JR, Koehl WF, Varley JB, Janotti A, Buckley BB, Van de Walle CG, Awschalom DD. Quantum computing with defects. P Natl A Sci 2010;107:8513-8.

[19] Milburn GJ. Quantum measurement and control of single spins in diamond. Science 2010;330:1188-9.

[20] Mita Y. Change of absorption spectra in type-i $b$ diamond with heavy neutron irradiation. Phys Rev B 1996;53:11360-4.

[21] Iakoubovskii K, Adriaenssens GJ, Nesladek M. Photochromism of vacancy-related centres in diamond. J Phys: Cond Matt 2000;12:189-99.

[22] Redman DA, Brown S, Sands RH, Rand SC. Spin dynamics and electronic states of $n-V$ centres in diamond by epr and fourwave-mixing spectroscopy. Phys Rev Lett 1991;67:3420-3.

[23] Felton S, Edmonds AM, Newton ME, Martineau PM, Fisher D, Twitchen DJ. Electron paramagnetic resonance studies of the neutral nitrogen vacancy in diamond. Phys Rev B 2008;77:081201.

[24] Neumann P, Kolesov R, Jacques V, Beck J, Tisler J, Batalov A, Rogers L, Manson NB, Balasubramanian G, Jelezko F, Wrachtrup J. Excited-state spectroscopy of single nv defects in diamond using optically detected magnetic resonance. New $\mathbf{J}$ Phys 2009;11:013017.

[25] Fuchs GD, Dobrovitski VV, Hanson R, Batra A, Weis CD, Schenkel T, Awschalom DD. Excited-state spectroscopy using single spin manipulation in diamond. Phys Rev Lett 2008;101:117601.

[26] Fu K-MC, Santori C, Barclay PE, Rogers LJ, Manson NB, Beausoleil RG. Observation of the dynamic jahn-teller effect in the excited states of nitrogen-vacancy centres in diamond. Phys Rev Lett 2009;103:256404.

[27] Maze JR, Gali A, Togan E, Chu Y, Trifonov A, Kaxiras E, Lukin MD. Properties of nitrogen-vacancy centers in diamond: the group theoretic approach. New J Phys 2011;13:025025.

[28] Acosta VM, Bauch E, Jarmola A, Zipp LJ, Ledbetter MP, Budker D. Broadband magnetometry by infrared-absorption detection of nitrogen-vacancy ensembles in diamond. Appl Phys Lett 2010;97:174104.

[29] Stacey A, Aharonovich I, Prawer S, and Butler JE. Controlled synthesis of high quality micro/nano-diamonds by microwave plasma chemical vapor deposition. Diam Rel Mat 2009;18:51-5.

[30] Pauzauskie PJ, Crowhurst JC, Worsley MA, Laurence TA, Kilcoyne ALD, Wang Y, Willey TM, Visbeck KS, Fakra SC, Evans WJ, Zaug JM, Satcher JH. Synthesis and characterization of a nanocrystalline diamond aerogel. P Natl A Sci 2011;108:8550-3.

[31] Pezzagna S, Naydenov B, Jelezko F, Wrachtrup J, Meijer J. Creation efficiency of nitrogen-vacancy centres in diamond. New J Phys 2010;12:065017.

[32] Pezzagna S, Wildanger D, Mazarov P, Wieck AD, Sarov Y, Rangelow I, Naydenov B, Jelezko F, Hell SW, Meijer J. Nanoscale engineering and optical addressing of single spins in diamond. Small 2010;6:2117-21.

[33] Tisler J, Balasubramanian G, Naydenov B, Kolesov R, Grotz B, Reuter R, Boudou J-P, Curmi PA, Sennour M, Thorel A, Boì sch M, Aulenbacher K, Erdmann R, Hemmer PR, Jelezko F, Wrachtrup J. Fluorescence and spin properties of defects in single digit nanodiamonds. ACS Nano 2009;3:1959-65.
[34] Smith BR, Inglis DW, Sandnes B, Rabeau JR, Zvyagin AV, Gruber D, Noble CJ, Vogel R, Åňsawa E, Plakhotnik T. Fivenanometer diamond with luminescent nitrogen-vacancy defect centres. Small 2009;5:1649-53.

[35] Smith BR, Gruber D, Plakhotnik T. The effects of surface oxidation on luminescence of nano diamonds. Diam Rel Mat 2010;19:314-8.

[36] Bradac C, Gaebel T, Naidoo N, Sellars MJ, Twamley J, Brown LJ, Barnard AS, Plakhotnik T, Zvyagin AV, Rabeau JR. Observation and control of blinking nitrogenvacancy centres in discrete nanodiamonds. Nat Nanotech 2010;5:345-9.

[37] Gaebel T, Domhan M, Popa I, Wittmann C, Neumann P, Jelezko F, Rabeau J, Stavrias N, Greentree A, Prawer S, Meijer J, Twamley J, Hemmer P, Wrachtrup J. Room-temperature coherent coupling of single spins in diamond. Nat Phys 2006;2:408-13.

[38] Aharonovich I, Castelletto S, Simpson DA, Su C-H, Greentree AD, Prawer S. Diamond-based single-photon emitters. Rep Prog Phys 2011;74:076501.

[39] Wrachtrup J, Jelezko F. Processing quantum information in diamond. J Phys: Cond Matt 2006;18:S807.

[40] Childress L, Gurudev Dutt MV, Taylor JM, Zibrov AS, Jelezko F, Wrachtrup J, Hemmer PR, Lukin MD. Coherent dynamics of coupled electron and nuclear spin qubits in diamond. Science 2006;314:281-5.

[41] Balasubramanian G, Neumann P, Twitchen D, Markham M, Kolesov R, Mizuochi N, Isoya J, Achard J, Beck J, Tissler J, Jacques V, Hemmer PR, Jelezko F, Wrachtrup J. Ultralong spin coherence time in isotopically engineered diamond. Nat Mat 2009;8:383-7.

[42] McGuinness LP, Yan Y, Stacey A, Simpson DA, Hall LT, Maclaurin D, Prawer S, Mulvaney P, Wrachtrup J, Caruso F, Scholten RE, Hollenberg LCL. Quantum measurement and orientation tracking of fluorescent nanodiamonds inside living cells. Nat Nanotech 2011;6:358-63.

[43] Lawson SC, Fisher D, Hunt DC, Newton ME. On the existence of positively charged single-substitutional nitrogen in diamond. J Phys: Cond Matt 1998;10:6171-80.

[44] Dumeige Y, Treussart F, Allamaume R, Gacoin T, Roch J-F, Grangier P. Photoinduced creation of nitrogen-related color centres in diamond nanocrystals under femtosecond illumination. J Lum 2004;109:61-7.

[45] Gaebel T, Domhan M, Wittmann C, Popa I, Jelezko F, Rabeau J, Greentree A, Prawer S, Trajkov E, Hemmer P, Wrachtrup J. Photochromism in single nitrogen-vacancy defect in diamond. Appl Phys B: Las Opt 2006;82:243-6.

[46] Manson N, Harrison J. Photo-ionization of the nitrogen-vacancy centre in diamond. Diam Rel Mat 2005;14:1705-10.

[47] Zheng D. Study and manipulation of photoluminescent nv color centre in diamond. PhD Dissertation, 2010.

[48] Wee T-L, Tzeng Y-K, Han C-C, Chang H-C, Fann W, Hsu J-H, Chen K-M, Yu Y-C. Two-photon excited fluorescence of nitrogen-vacancy centres in proton-irradiated type ib diamond. J Phys Chem A 2007;111:9379-86.

[49] Lin C-K, Wang Y-H, Chang H-C, Hayashi M, Lin SH. Oneand two-photon absorption properties of diamond nitrogenvacancy defect centres: a theoretical study. J Chem Phys 2008;129:124714.

[50] Trojánek F, Žídek K, Dzurňák B, Kozák M, Malý P. Nonlinear optical properties of nanocrystalline diamond. Opt Expr 2010; 18:1349-57.

[51] Redman D, Shu Q, Lenef A, Rand SC. Two-beam coupling by nitrogen-vacancy centres in diamond. Opt Lett 1992;17: $175-7$. 
[52] Degen CL. Scanning magnetic field microscope with a diamond single-spin sensor. Appl Phys Lett 2008;92:243111.

[53] Takahashi S, Hanson R, van Tol J, Sherwin MS, Awschalom DD. Quenching spin decoherence in diamond through spin bath polarization. Phys Rev Lett 2008;101:047601.

[54] Maertz BJ, Wijnheijmer AP, Fuchs GD, Nowakowski ME, Awschalom DD. Vectormagnetic field microscopy using nitrogen vacancy centres in diamond. Appl Phys Lett 2010;96:092504.

[55] Steinert S, Dolde F, Neumann P, Aird A, Naydenov B, Balasubramanian G, Jelezko F, Wrachtrup J. High sensitivity magnetic imaging using an array of spins in diamond. Rev Sci Instrum 2010;81:043705.

[56] Stanwix PL, Pham LM, Maze JR, Le Sage D, Yeung TK, Cappellaro P, Hemmer PR, Yacoby A, Lukin MD, Walsworth RL. Coherence of nitrogen-vacancy electronic spin ensembles in diamond. Phys Rev B 2010;82:201201.

[57] Lai ND, Zheng D, Jelezko F, Treussart F, Roch J-F. Influence of a static magnetic field on the photoluminescence of an ensemble of nitrogen-vacancy color centres in a diamond single-crystal. Appl Phys Lett 2009;95:133101.

[58] Pham LM, Sage DL, Stanwix PL, Yeung TK, Glenn D, Trifonov A, Cappellaro P, Hemmer PR, Lukin MD, Park H, Yacoby A, Walsworth RL. Magnetic field imaging with nitrogen-vacancy ensembles. New J Phys 2011;13:045021.

[59] Pendry JB. Negative refraction makes a perfect lens. Phys Rev Lett 2000;85:3966-9.

[60] Tzeng Y-K, Faklaris O, Chang B-M, Kuo Y, Hsu J-H, Chang $\mathrm{H}-\mathrm{C}$. Superresolution imaging of albumin-conjugated fluorescent nanodiamonds in cells by stimulated emission depletion. Angew Chem Int Ed 2011;50:2262-5.

[61] Hell SW, Wichmann J. Breaking the diffraction resolution limit by stimulated emission: stimulated-emission-depletion fluorescence microscopy. Opt Lett 199419:780-2.

[62] Harke B, Keller J, Ullal CK, Westphal V, Schönle A, Hell SW. Resolution scaling in STED microscopy. Opt Express 2008; 16:4154-62.

[63] Chang Y-R, Lee H-Y, Chen K, Chang C-C, Tsai D-S, Fu C-C, Lim T-S, Tzeng Y-K, Fang C-Y, Han C-C, Chang H-C, Fann W. Mass production and dynamic imaging of fluorescent nanodiamonds. Nat Nanotech 2008;3:284-8.

[64] Wildanger D, Maze JR, Hell SW. Diffraction unlimited alloptical recording of electron spin resonances. Phys Rev Lett 2011;107:017601.

[65] Maurer PC, Maze JR, Stanwix PL, Jiang L, Gorshkov AV, Zibrov AA, Harke B, Hodges JS, Zibrov AS, Yacoby A, Twitchen D, Hell SW, Walsworth RL, Lukin MD. Far-field optical imaging and manipulation of individual spins with nanoscale resolution. Nat Phys 2010;6:912-8.

[66] Koehl WF, Buckley BB, Heremans FJ, Calusine G, Awschalom DD. Room temperature coherent control of defect spin qubits in silicon carbide. Nature 2011;479:84-7.

Received January 5, 2012; accepted May 18, 2012 